\title{
Ecología de dispersión y reclutamiento de bosques húmedos tropicales.
}

\author{
Javier Ruiz \\ Programa Científico Complementario (PCC)
}

Se presenta una síntesis con los avances científicos alcanzados por un programa de investigación ecológica en el campo de los efectos de las interacciones biologías en la dinámica de reclutamiento de bosques húmedos tropicales. Este programa de investigación inició en el año 2002, como parte de una nueva fase de estudio de la ecología de regeneración del bosque húmedo tropical del sureste de Nicaragua (Ruiz et al. 2008). En esta comunicación se presentan resultados alcanzados durante el proceso de prueba de dos hipótesis: (1) la hipótesis de limitación de reclutamiento y (2) la hipótesis de Janzen-Connell; estas dos hipótesis se enfocan en comprender el proceso de organización de bosques húmedos tropicales (Ruiz 2008). En específico evaluamos la importancia relativa de dispersión de semillas en la sobrevivencia de semillas y plántulas. En el proceso de prueba de hipótesis estudiamos la especie $D$. oleifera.

Paralelamente planteamos objetivos académicos enfocados en el fortalecimiento de las instituciones universitarias nicaragüenses. Para ello, a la par de las investigaciones científicas se ha priorizado el desarrollo intelectual de estudiantes universitarios nicaragüenses, con énfasis en los estudiantes de las Regiones Autónomas de la Costa Caribe. En la actualidad se están desarrollando protocolos de investigación con el objetivo de localizar fondos para continuar mejorando las ciencias biológicas en Nicaragua y el sistema universitario nacional, a través de la complementación de la capacitación del recurso humano. La presente comunicación es parte de una serie de artículos que tienen como objetivos contribuir al proceso de popularización de las ciencias ecológicas nicaragüenses y el fortalecimiento de la ciencias naturales en Nicaragua (Ruiz et al. 2008).

El área general de estudio es el área dañada por el huracán Juana en 1988. El bosque en estudio forma parte del bosque húmedo siempre verde de bajura según el sistema de clasificación de zonas de vida de Holdridge (1992). La temperatura media anual es de $26^{\circ} \mathrm{C}$, evaporación anual 1,332.4 mm (INDERA 1991). La vegetación es característica de bosque húmedo tropical del tipo que se extiende desde Centro América, Belice y Panamá. El estudio se realizó en una parcela permanente de 6.5 hectáreas establecida en el sitio La Unión (Lat.12 ${ }^{\circ} 5^{\prime} \mathrm{N}$. $83^{\circ} 53^{\prime}$ W.). Estudiamos la especie D. oleifera la cual se encuentra presente en Centro América y Venezuela. Sus árboles pueden alcanzar hasta 50 metros de altura, la madera es muy dura y se utiliza para fabricar carbón, postes de cercas y las nueces se utilizan como suplemento alimenticio.

\section{Dinámica de dispersión y reclutamiento de plantas}

La producción de semillas, dispersión de semillas y el reclutamiento de plántulas son procesos importantes en la dinámica de poblaciones, precisamente por que éstos casi nunca son completamente exitosos (Clark et al. 1999a; Muller-Landau et al. 2002). Primero, la falta de arribo de semillas a todos los sitios del sotobosque limita las tasas de crecimiento poblacional, abundancia y distribución, un fenómeno denominado como limitación de semillas. La limitación de semillas tiene importantes consecuencias para la dinámica de población y comunidad, así como para la diversidad de especies a nivel local y regional (Hurtt y Pacala 1995; Tilman 1994). La limitación de semillas puede surgir de la baja producción de semillas o debido a la baja dispersión de las semillas presentes, incluso, cuando las semillas se encuentran localmente abundantes. El número de sitios, en el bosque, que pueden ser posiblemente alcanzados por las semillas depende de la abundancia local de árboles adultos y de su fertilidad, lo cual determina la densidad total [acumulada] de semillas (Clark et al. 1998). La varianza en la densidad de semillas depende primariamente de la forma y tamaño de la distribución de semillas, las cuales indican cómo se encuentran distribuidas con respecto al adulto congénere más cercano (Clark et al. 1998). Cuando los árboles se encuentran agrupados, como frecuentemente se les encuentra (Condit et al. 2000), la 


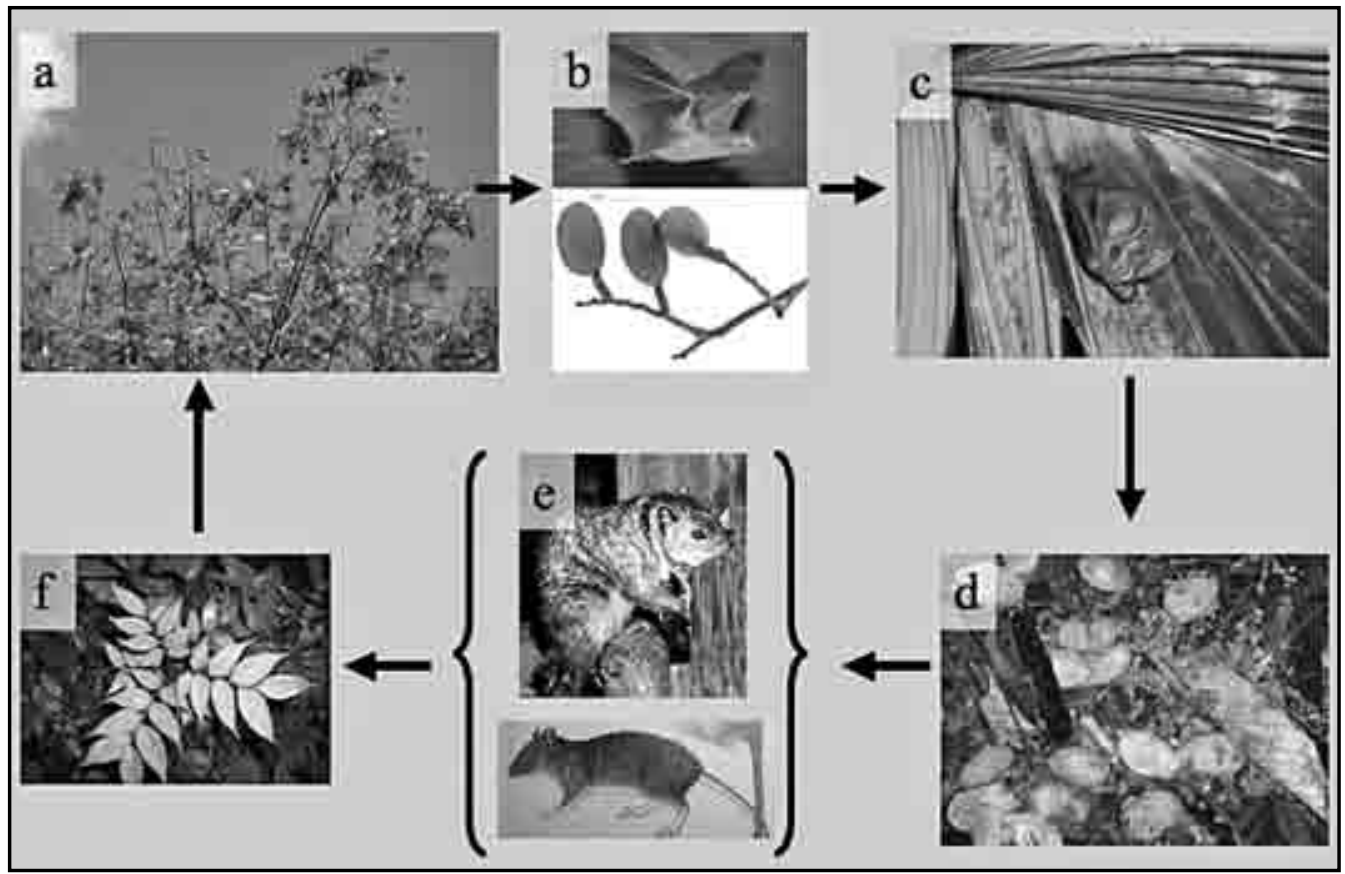

Figura 1. Esquematización del proceso de dispersión de D. oleifera. El árbol en fructificación " $a$ ", los frutos son transportados por murciélagos " $b$ ", hacia palmas usadas como comederos " $c$ ". Los murciélagos consumen el fruto y dejan caer las semillas en congregaciones de semillas " $d$ ", las cuales son localizadas por mamíferos terrestres " $e$ ". Los mamíferos terrestres consumen unas semillas y dispersan otras, dando lugar al reclutamiento de plántulas " $f$ ". Fotografías de Delvis Guillen, Javier Ruiz y John Vandermeer; ilustración de Reid (1997). congéneres establecidos (Janzen 1970). Un estudio de campo del reclutamiento de plántulas realizado en la Isla de Barro Colorado (Panamá) ha presentado evidencia concreta en favor de la hipótesis de Janzen-Connell a nivel de la comunidad de plantas (Condit et al. 1992). Aunque es muy poco loque se conoce acerca de la dinámica ecológica responsable por los patrones de JanzenConnell observados en el campo. Es por ello que en el presente artículo se evalúa la hipótesis de Janzen-Connell estudiando la distribución, dispersión, el consumo de semillas, reclutamiento de plántulas y herbivoría insectívora de la especie $D$. oleifera. Es decir, además del estudio de las distribuciones de semillas y plántulas se evalúan las interacciones limitación de semillas será mucho mayor (Ribbens et al. 1994). Los incrementos en la varianza de la densidad de semillas se pueden observar en el campo como agregados de semillas localizadas en el sotobosque.

Una hipótesis importante acerca de la dinámica del reclutamiento es la de Janzen (1970) y Connell (1971). La proposición básica de esta hipótesis es que los enemigos naturales de las plantas, como los insectos herbívoros, serán responsable de mayor daño debido a que éstos responden positivamente a la abundancia de propágulos cerca al árbol huésped. Es por ello que escapar a los efectos adversos de los enemigos naturales es crucial para el éxito de la demografía de plantas (Howe y Smallwood 1982). El incremento en la dispersión de semilla potencialmente reduce los efectos negativos asociados con la densidad de propágalos cerca al árbol congénere más cercano. De esta forma, el patrón de sobreviviencia de semillas y reclutamiento de plántulas dará como resultado largas distancias medias entre plántulas y el árbol congénere más cercano (Blundell y Peart 1998; Clark et al. 1999b; Condit et al. 1992; Hyatt et al. 2003). Consecuentemente, el alto número de especies de árboles de bosque húmedo tropical podría estar coexistiendo en bajas densidades y con largas distancias entre árboles biológicas responsables de la dispersión de semillas y el reclutamiento de plántulas.

\section{La ecología de dispersión de semillas}

La ecología de regeneración sugiere que la acumulación de semillas bajo el árbol adulto conlleva a efectos negativos de densidad en los cuales los propágalos son encontrados con facilidad por insectos herbívoros, granívoros, etcétera - la esencia de la hipótesis de Janzen y Connell. Esta investigación se enfoca en comprender la dinámica de reclutamiento de semillas, plántulas y vástagos de $D$. oleifera, una especie común y representativa del bosque del sureste de Nicaragua. En esta fase, las hipótesis especificas que se evalúan son: "hipótesis de distribución de semillas", sí la dispersión de semillas es al azar, entonces, la distribución de semillas no es diferente de una distribución de Poisson. "Hipótesis de dispersión de semillas", sí existe una diferencia en importancia relativa ente la dispersión primaria y secundaria, entonces habrá mayor sobrevivencia de semillas después de la dispersión secundaria. Comprender los detalles de la dispersión de semillas conllevará a determinar el efecto que tiene la dispersión de semillas en la reducción de la tasa de 
mortalidad y el aumento en la tasa de sobrevivencia de especies de bosque húmedo tropical. Analizamos cada unas de las hipótesis planteadas aquí, usando una base de datos de alta resolución que incluye información acerca de la posición y demografía de semillas, plántulas, vástagos y árboles desde 2002 hasta 2006.

El proceso de dispersión de semillas de $D$. oleifera involucra la dispersión primaria y la dispersión secundaria. La dispersión primaria ocurre cuando las semillas son removidas del árbol -ejemplo, por murciélagos-o cuando las semillas caen bajo la copa del árbol reproductor por gravedad (Figura 1). Los murciélagos son dispersores primarios y vuelan hasta las ramas de los árboles para transportar las semillas agarradas entre sus garras. Supuestamente, los murciélagos tienen que volar y agarrar las semillas rápidamente para evitar ser depredados por sus enemigos naturales presente en las copas de los árboles, tales como las boas y búhos. Los murciélagos dispersan las semillas hacia sus comederos, los cuales son sitios donde éstos perchan para consumir la estructura carnosa que rodea la semilla. En un estudio de campo determinamos que las palmas son utilizadas por los murciélagos como comederos en el 79\% de los casos (Ruiz y Boucher 2009). Investigadores de campo consideran que esta preferencia por las palmas es debido en parte a que las estructuras de algunas de las hojas de las palmas -ejemplo, Cryosophila warsewitzii- protegen a los murciélagos de la lluvia y de los enemigos naturales presente en los doseles superiores del bosque. Cuando los murciélagos perchan en las palmas, éstos remueven una porción del tejido de las hojas, a través de los cuales se sostienen de sus garras y se posan boca abajo (Figura 1c). Las especies de murciélagos capturadas durante el muestreo de murciélago son: Artibeus intermedius, Artibeus jamaicensis, Artibeus lituratus, Artibeus phaeotis, Artibeus watsoni, Carolia perspicillata, Chiroderma villosum, Carolia castanea, Myotis nigricans, Phyllostomus discolor, Trachops cirrhosus, Vampyressa nymphaea, Glossophaga soricina y Hylonycteris underwoodi. Los murciélagos del género Artibeus son potencialmente dispersores de semilla de D. oleifera y de otras especies de semilla grande, puesto que el tamaño de murciélagos de este género son lo suficientemente grandes para dispersar semillas de varios tamaños. Las identificaciones taxonómicas de los murciélagos fueron realizadas por Jason Beck y Stephanie Leslie de la Universidad de Utah.

Luego de consumir la estructura carnosa de las semillas, éstas son dejadas caer una tras otra hasta que forman un montículo bajo la hoja donde los murciélagos se encuentran perchando. Algunas veces, las concentraciones de semillas son de 30 a 40 semillas, pero normalmente se les encuentra en número rodeando las 20 semillas (Figura 1d). Estas concentraciones de semillas son generalmente encontradas a unos 20 a 30 metros de distancia del árbol congénere más

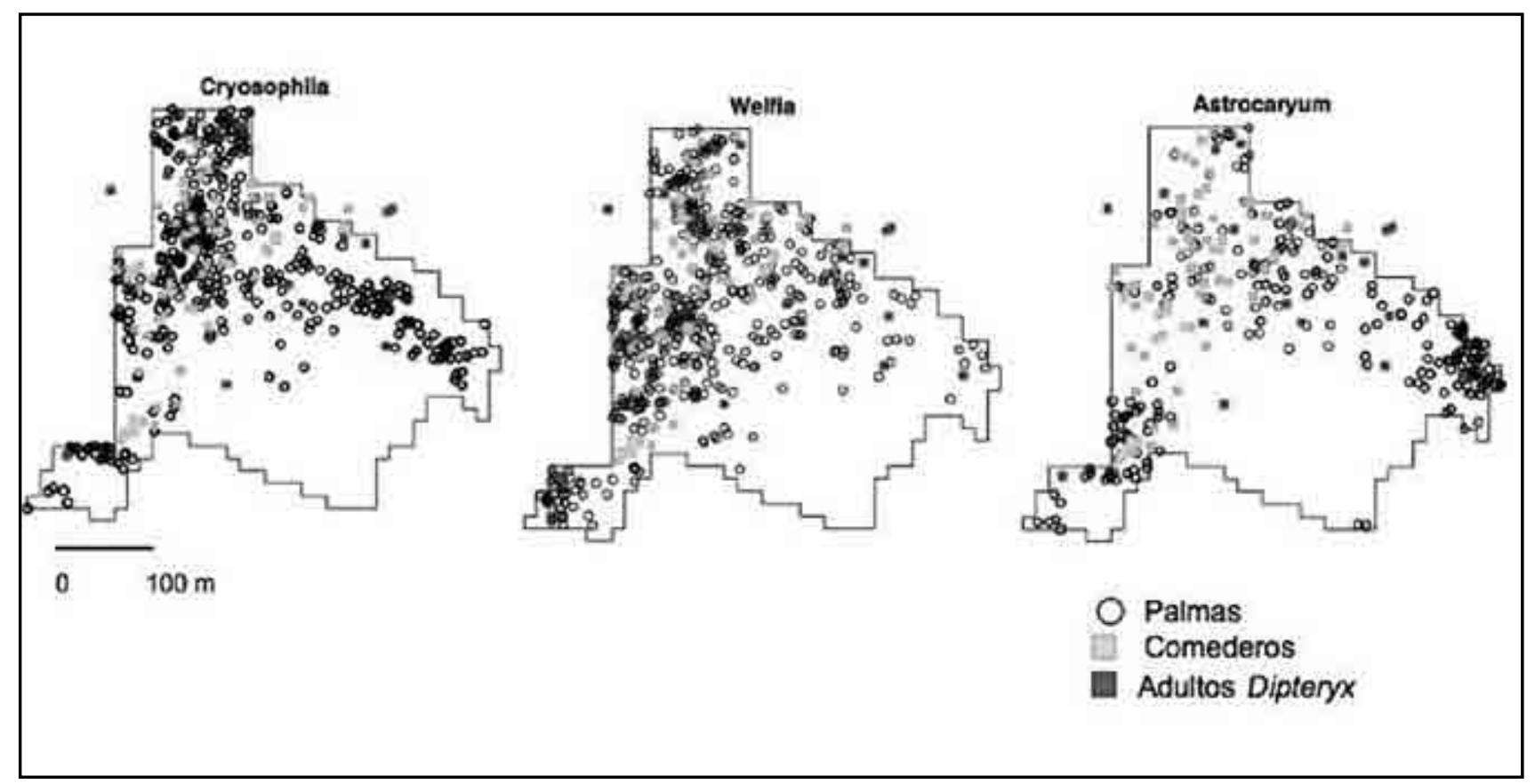

Figura 2. Distribución de las tres especies de palmas más frecuentemente utilizada por murciélagos para establecer sus comederos. Los murciélagos seleccionaron con mayor frecuencia a las palmas Cryosophila y Welfia, localizadas lejos de árbol D. oleifera, donde la sobrevivencia de plántulas es mayor (Ruiz y Boucher 2009). 
cercano. Es altamente probable que los murciélagos estén seleccionando palmas localizadas lejos y no cerca del árbol de D. oleifera más cercano, para escapar de la depredación por boas y búhos. Arribamos a esta conclusión luego de observar y comparar las distribuciones de las tres especies de palmas que son más usadas por los murciélagos para perchar y la distribución de las palmas utilizadas como comederos en 2005. La posición de las palmas utilizadas como sitios comederos de murciélagos fueron aquellas que se encontraban a varias decenas de metros del árbol de $D$. oleifera más cercano, y no las palmas que se encontraban cerca de éstos (Figura 2, Ruiz y Boucher 2009).

Un problema fundamental para el reclutamiento es que después de la dispersión primaria las semillas se encuentran en altas densidades -incluso mucho más agrupadas que debajo del árbol reproductivo- y por ello podrían ser encontradas con facilidad por los consumidores de semillas. Entonces es necesario que las semillas sean dispersadas lejos de estas congregaciones de semillas. Los vertebrados terrestres, tales como ardillas y guardatinajas, son los encargados de la dispersión secundaria de semillas (Figura 1e). A la fecha, las especies de vertebrados terrestres capturados durante los estudios de campo en el sitio de estudio son: Proechimys semispinous, Oryzomys spp., Nyctomys sumichirasti, Marmosa mexicana y Didelphis virginiana. Las identificaciones taxonómicas de los mamíferos terrestres fueron realizadas por Delvis Guillen de las Universidades de las Regiones Autónomas de Nicaragua (URACCAN). Los vertebrados terrestres buscan las semillas en el sotobosque, no está claro sí utilizan el olfato, la memoria o ambos sentidos para encontrar las congregaciones de semillas. Es interesante el hecho que el papel de estos dispersores secundarios es dual, puesto que actúan como consumidores de semillas y dispersores secundarios (Vander Wall 2005). La dispersión secundaria ocurre cuando las semillas son removidas de las concentraciones hacia otros sitios del sotobosque. Posiblemente para esconderlas de competidores en agrupaciones llamadas cashes y luego esparcirlas en el sotobosque, ya que algunas veces la ubicación de semillas es olvidada, éstas son dispersadas secundariamente (DeMattia et al. 2004; Ruiz 2008).

\section{Contribución relativa de la dispersión primaria y secundaria para la sobrevivencia de semillas}

Los resultados de las investigaciones de campo presentan evidencia en favor de la idea que la dispersión secundaria es fundamentalmente necesaria para escapar de los efectos negativos de densidad, específicamente, la granivoría. Las observaciones de campo muestran que la dispersión primaria redujo la mortalidad de semillas, aproximadamente $70 \%$, a razón de $1.2 \%(\mathrm{p}<.001)$ por cada metro que la semilla fue movida con respecto al árbol congénere más cercano. La dispersión secundaria por su parte redujo la probabilidad de mortalidad en 24\% ( $p<.001$ ) (Ruiz 2008, 2009). La dispersión secundaria ocurre con mayor frecuencia lejos del árbol $D$. oleifera, principalmente debido a que los murciélagos dispersaron primariamente semillas bajo las palmas localizadas lejos del árbol congénere más cercano. Además, que los mamíferos terrestres no movieron semillas cerca al árbol reproductivo, probablemente debido a la presencia de sus enemigos naturales (boas y serpientes) presente en las bases de los árboles de D. oleifera. De esta forma, el papel conjugado de los murciélagos y mamíferos terrestres es fundamentalmente necesario para el proceso de dispersión y sobrevivencia de semillas de $D$. oleifera. Los patrones de dispersión primaria y secundaria observados en nuestra investigaciones son comunes en otras especies de bosques húmedos tropicales (Forget y Wenny 2005). Los resultados indican que, después de la dispersión primaria y secundaria, una proporción de las semillas se encuentra lejos del árbol de D. oleifera más cercano.

Después del proceso de dispersión secundaria, una proporción de las semillas se encuentra en áreas de baja densidad. Del total de semillas producidas por los adultos de D. oleifera, aproximadamente $5 \%$ son dispersadas lejos del árbol congénere más cercano (Ruiz 2008, 2009). Por lo tanto, a baja densidad y en mejores condiciones para el reclutamiento de plántulas. En la próxima fase del proceso de la dispersión de plantas, las preguntas de investigación han girado en torno a: ¿cuál es la distribución espacial del reclutamiento de plántulas y estadios de mayor tamaño? Por un lado, la "hipótesis de reclutamiento" (Clark et al. 1999a; Clark et al. 1999b) -parte de la teoría de ensamblaje de dispersión de comunidades de Hubbell (2001)-propone que la probabilidad de reclutamiento de plántulas es igual en cualquier punto del bosque. La hipótesis alternativa es la hipótesis de Janzen y Connell, la cual enuncia que debido a la importancia de la presión constante de los enemigos naturales, la regeneración de plántulas ocurre lejos de las concentraciones de semillas; donde los enemigos naturales encuentran con menor frecuencia las semillas y las plántulas.

\section{Ecología del reclutamiento de plántulas}

Esta fase de la investigación se enfocó en determinar la dinámica de reclutamiento de plántulas y vástagos y hasta qué punto la herbivoría insectívora es responsable del 
reclutamiento de D. oleifera en el sureste de Nicaragua. Se considera herbivoría insectívora el consumo de las partes vivas pero no vitales de las plantas por insectos. La herbivoría foliar es responsable de cerca del 10 por ciento del daño a hojas en bosques húmedos tropicales (Dirzo 1987). La mayoría de este daño es causado por larvas de mariposas (Coley y Barone 1996; Coley 1990; Dirzo 1987). Este tipo de daño puede afectar negativamente la demografía de plántulas (Coley 1983; Coley 1990; Dirzo 1987) y puede ser lo suficientemente severa para influenciar la dinámica de demografía de plántulas (Sullivan 2003). Las hipótesis especificas que estamos probando son: la "hipótesis de reclutamiento de plántulas"; sí la distribución del reclutamiento de plántulas es mayor que la esperada al azar, entonces, la distribución del reclutamiento es agrupado. "Hipótesis de escape de enemigos naturales"; sí el daño de herbivoría insectívora en plántulas decrece con la distancia al árbol congénere más cercano, entonces, la sobrevivencia de plántulas estará ubicada a mayor distancia de éstos. En otras palabras, las bajas tasas de daño de herbivoría insectívora estarán positivamente correlacionadas con las altas proporciones de sobrevivencia

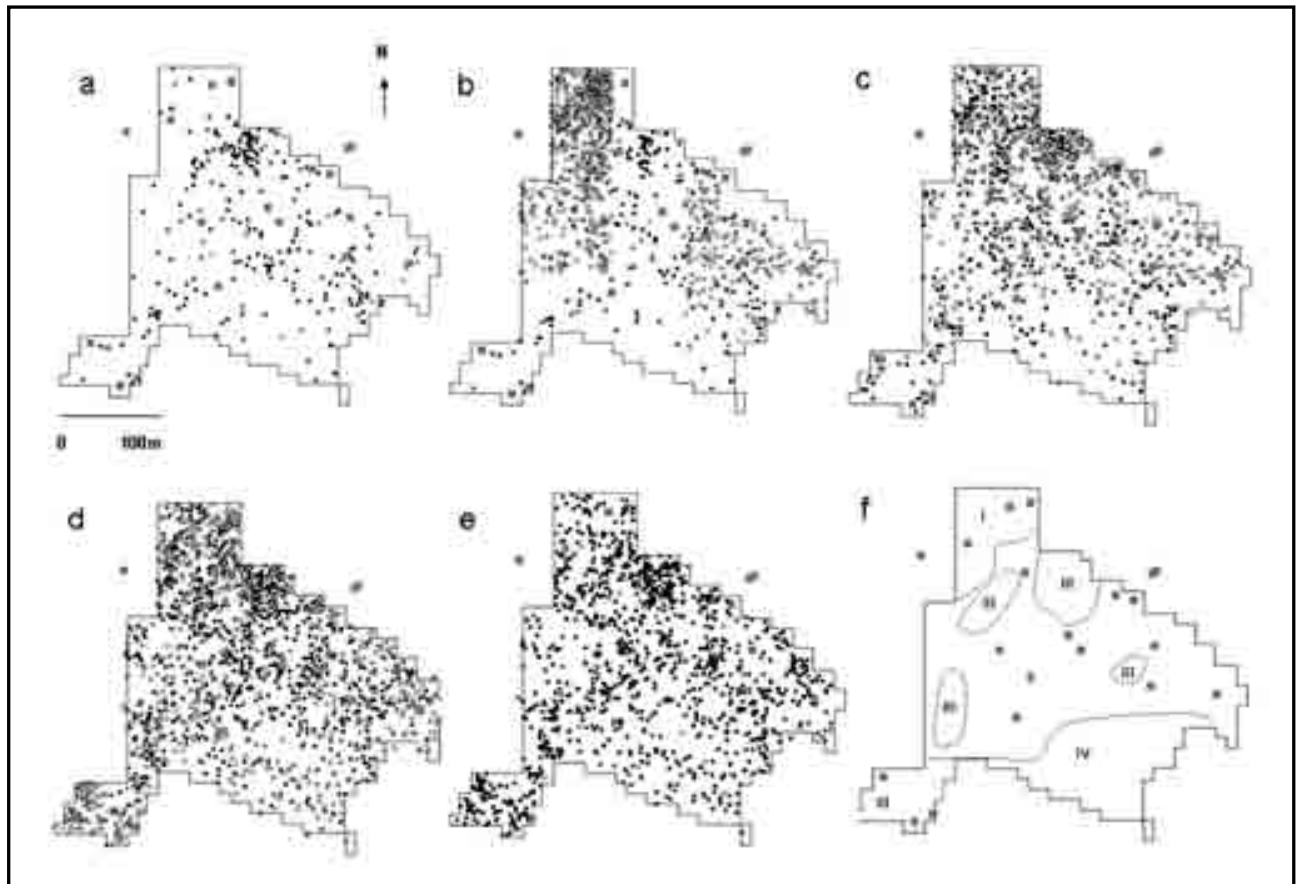

Figura 3. Distribución del reclutamiento de plántulas de D. oleifera. Cuadros grises representan la posición de árboles adultos, círculos sólidos representan reclutamiento de plántulas por más de un año y los círculos blancos corresponden al reclutamiento de plántulas por menos de un año. Las letras de " $a-e$ " corresponden a los datos de 2002-2006 y " $f$ " es un diagrama de las áreas donde el reclutamiento de plántulas es en parches. I) parches con gran número de plántulas cerca a árboles adultos; II) parches con bajo número de plántulas lejos del árbol adulto; III) parches con alto número de plántulas y IV) con bajo número de plántulas (Ruiz et al. 2009b). de plántulas ubicadas lejos del árbol congénere más cercano. Complementariamente a esta hipótesis está el determinar sí la tasa de crecimiento relativa se incrementa como una función dependiente de la distancia al árbol congénere más cercano. Sí éste es el caso, entonces habrá evidencia que sugerirá una correlación positiva entre las áreas con baja tasas de herbivoría insectívora y altas tasas de crecimiento de las plántulas. "Hipótesis de reclutamiento de vástagos"; sí la distribución del reclutamiento de vástagos es agrupada, entonces, esta distribución será diferente a una distribución al azar. Otra hipótesis es determinar sí la proporción de la mortalidad de plántulas está negativamente correlacionada con el número de vástagos; entonces existirá evidencia que sugiere una correlación positiva entre la presencia de enemigos naturales y el número de vástagos. Proponemos que los enemigos naturales podrían estar utilizando a los vástagos para encontrar a las plántulas.

La distribución de sobrevivencia de plántulas de D. oleifera es consistente con el patrón esperado por la hipótesis de Janzen-Connell. Nuestras observaciones de campo muestran que el patrón Janzen-Connell de reclutamiento de plántulas es influenciado por la herbivoría insectívora. En un estudio de campo, los niveles de daño de herbivoría insectívora fueron muy altos cerca del árbol congénere más cercano, donde había alta densidad de plántulas (Clark y Clark 1985). El patrón espacial del daño de herbivoría reportado en nuestro estudio del reclutamiento de plántulas de D. oleifera en Nicaragua presenta evidencia en favor de la "hipótesis de escape de los enemigos naturales" (Ruiz et al. 2009a). Las técnicas de estadística espacial de la dinámica del proceso de reclutamiento agrega un componente temporal y espacial en el proceso de comprender cómo el reclutamiento ocurre en bosques húmedos tropicales (Ruiz et al. 2009b). Debido a que el efecto de la herbivoría insectívora es menos importante en el reclutamiento de vástagos, juveniles y árboles se ha propuesto la idea alternativa 
que las condiciones de micrositio, específicamente las condiciones de luz arriba de las plántulas y estadios de mayor tamaño, estarán positivamente correlacionadas con el tamaño de la copa, tronco y el crecimiento (Clark y Clark 1985). Un resultado importante en esta investigación de campo es que la formaciones de claros de luz es independiente de la posición del árbol de D. oleifera (Clark y Clark 1987), lo cual sugiere que la dinámica del reclutamiento de los estadios de mayor tamaño sería inconsistente con el modelo de Janzen-Connell. Nuestras observaciones de campo en el bosque húmedo tropical de Nicaragua sugiere que el reclutamiento de vástagos y juveniles podría estar correlacionado con la formación de claros de luz formados al azar (Ruiz 2008, 2009), tal y como ha sido reportado con anterioridad (Clark y Clark 1987). Para especies con bajo nivel de limitación de dispersión y reclutamiento, la formación de claros de luz al azar resultaría ventajosa para su regeneración. Específicamente, debido a que la especie D. oleifera se encuentra presente en un 90 por ciento del área de estudio, ésta tendría un mayor "chance" de reclutar en el dosel del bosque una vez que se forme un claro de luz al azar.

\section{Ecología del establecimiento de plántulas y vástagos}

Las observaciones a largo plazo de la dinámica de reclutamiento de $D$. oleifera indican que la distribución del reclutamiento de plántulas ocurre en parches y la transición de plántulas a vástagos es al azar (Ruiz et al. 2009b). La distribución del reclutamiento de plántulas se encuentra en parches localizados lejos del árbol congénere más cercano (Figura 3); este patrón es el resultado de la dispersión de semillas por murciélagos y mamíferos terrestres. Por otro lado, el patrón de reclutamiento de vástagos podría ser debido a las condiciones de luz en el sotobosque producto de la formación de micro-claros de luz cuando un árbol poda una rama o un árbol muere en el dosel del bosque. Los resultados de las observaciones de las distribuciones del reclutamiento de plántulas muestran que ésta ocurre lejos del árbol congénere más cercano, donde la densidad de plántulas y la incidencia de la herbivoría insectívora son menores.

En dos estudios de campo se ha presentado evidencia que sugiere que inclusive bajos niveles de herbivoría insectívora son suficientes para causar mortalidad de plántulas (Clark y Clark 1985; Ruiz et al. 2009b). Las evidencias de las curvas de sobrevivencia de plántulas con respecto al árbol congénere más cercano (Figura 4) y las funciones de herbivoría insectívora con respecto al árbol congénere más cercano indican una correlación entre las tasas bajas de herbivoría insectívora y la sobrevivencia de plántulas (Figura 5). Aunque los resultados de las observaciones muestran que la tasa de crecimiento de plántulas es independiente a su posición con respecto al árbol congénere más cercano. Este patrón de crecimiento podría ser producto de que las plántulas se encuentran cerca del punto de compensación de la luz -donde la respiración iguala la fotosíntesis. Los resultados presentados aquí sugieren que ambas hipótesis, la hipótesis de Janzen-Connell y la hipótesis de limitación de reclutamiento son operativas en diferentes estadios de desarrollo de las plantas. Durante los estadios iniciales, la dispersión de semillas y los insectos herbívoros determinan la sobrevivencia de semillas y plántulas. Los claros de luz formados al azar determinan la organización del reclutamiento de vástagos.

\section{Efecto de las cadenas trófica en el establecimiento de plántulas}

Las especies en una comunidad se encuentran unidas directa o indirectamente por medio de la interacciones entre consumidores y productores -el clásico ejemplo son las comunidades bióticas de los lagos (Carpenter et al. 2001). En ecosistemas con tres niveles tróficos -como son plantas, herbívoros artrópodos y depredadores de insectos herbívoros- una cadena trofica describe el efecto de los depredadores en la abundancia, riqueza y la estructura de los productores (Gruner 2004; Hairston et al. 1960; Levins 1974; Moreau et al. 2006). Por ejemplo, arañas (Romero y Vasconcellos-Neto 2004), hormigas arbóreas (Floren et al. 2002) y pájaros (Van Bael et al. 2003) son depredadores importantes de artrópodos herbívoros y su efecto en la demografía de plántulas es frecuentemente asociado con la reducción de la abundancia de artrópodos (Van Bael y Brawn 2005; Van Bael et al. 2003) y herbivoría insectívora (Clark y Clark 1985), frecuentemente debido a la depredación de herbívoros artrópodos por arañas (Romero y Vasconcellos-Neto 2004). Una reducción en la herbivoría insectívora puede resultar en altas tasas de reclutamiento en especies de bosques húmedos del trópico, debido a que la herbivoría foliar reduce la productividad neta de las plantas (Clark et al. 1993; Coley 1983; Coley 1990; Coley et al. 1985).

En esta fase se cuantificó el efecto de las interacciones tróficas en la dinámica de sobrevivencia de plántulas de $D$. oleifera por medio de investigaciones de campo. En específico se cuantificó hasta qué punto la presencia de arañas en plántulas contribuye con la sobrevivencia por medio de una reducción en las tasas de herbivoría insectívora de plántulas. Los resultados indican que la 


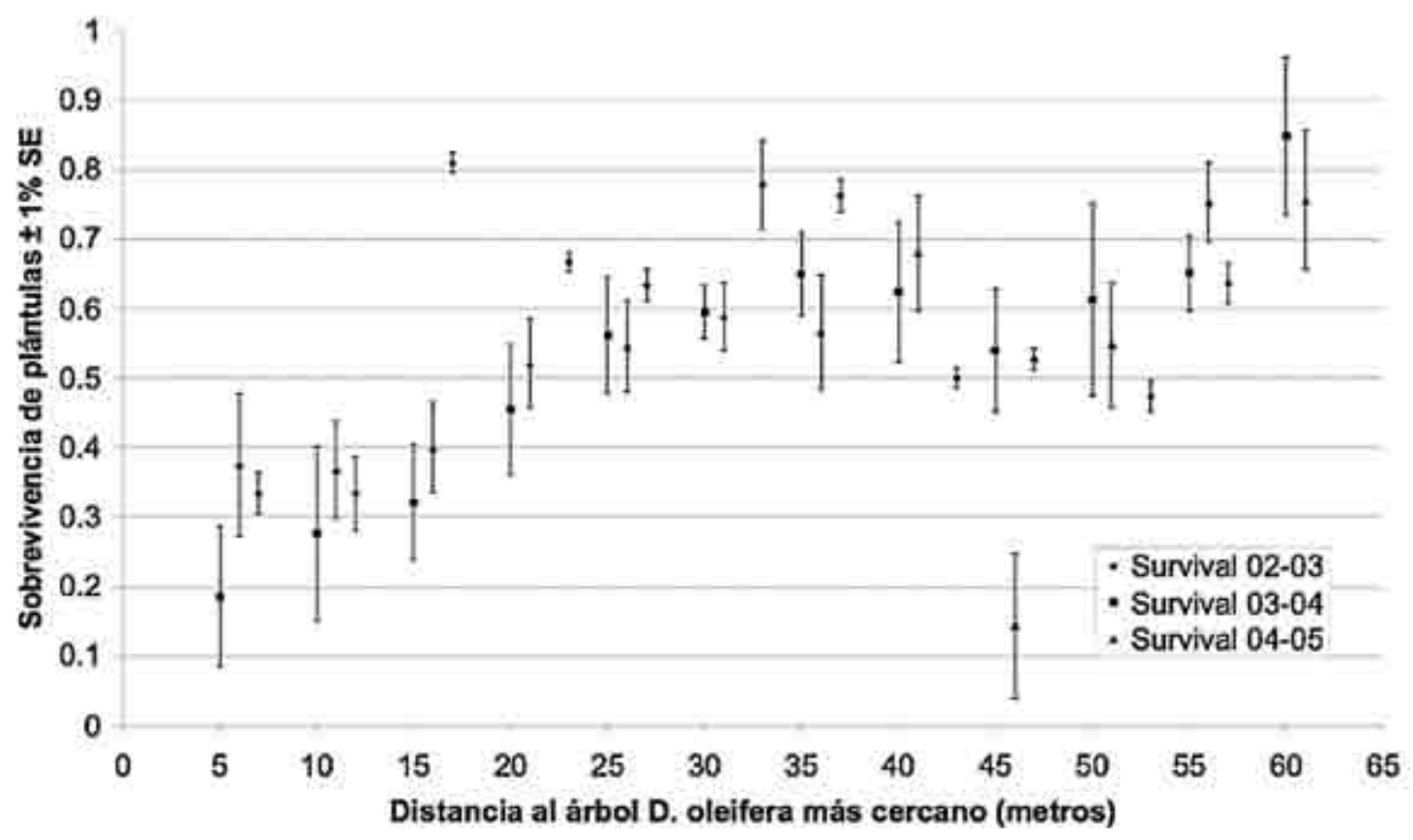

Figura 4. Sobrevivencia de plántulas de D. oleifera en relación a la distancia al árbol congénere más cercano (Ruiz 2008).

presencia de arañas se correlaciona significativamente con niveles bajos de herbivoría insectívora en plántulas de D. oleifera. El promedio ponderado de la herbivoría insectívora en plántulas que no tenían la presencia de arañas es aproximadamente $25 \%$ del total del área foliar. Por otro lado, las plántulas con presencia de arañas presentaron un daño de $15 \%$ del área foliar (Ruiz et al. 2009a). Aunque hasta el momento hemos determinado una correlación entre la presencia de arañas y bajos niveles de herbivoría insectívora, los detalles de las interacciones no se han cuantificado. Las observaciones en otros sistemas forestales indican que las arañas podrían estar depredando y/o acosando a los insectos herbívoros (Vandermeer et al. 2002). Ya que no se observaron insectos herbívoros durante los monitoreos diurnos, esta interacción podría estar ocurriendo durante la noche. Una línea de investigación de mucha relevancia actual sería determinar si la distribución espacial del reclutamiento de plántula se correlaciona con la presencia de arañas y la baja tasa de herbivoría insectívora.

Los resultados de nuestras observaciones en el sureste de Nicaragua muestran que el reclutamiento de plántulas ocurre en pequeños parches ubicados lejos del árbol de $D$. oleifera. Una concatenación de estas ideas sugiere que la presencia de arañas en dichos parches de plántulas podría estar reduciendo la herbivoría insectívora por medio de la depredación de insectos herbívoros dando así como resultado una alta sobrevivencia de plántulas -la posible explicación para las distribuciones agrupadas de plántulas (Figura 3). Estos aspectos de la presente investigación requieren mayor investigación, para lo cual se están sometiendo propuestas de investigación a revisión para su financiamiento y así poder realizar experimentos de campo que eluciden el papel de las arañas en la regeneración de especies de bosques húmedos tropicales. Por otro lado observamos que la transición de plántulas a vástagos es menos del $1 \%$ y la distribución del reclutamiento de vástagos es al azar. Los resultados muestran una correlación positiva entre altos niveles de herbivoría insectívora en plántulas que tuvieron vástagos cerca (Ruiz et al. 2009b). Es posible que la posición de los vástagos sirva como señal a los insectos herbívoros para así localizar la posición de las plántulas. Los resultados indican que es necesario determinar hasta qué punto la transición de plántulas a vástagos está determinada por la presencia de arañas y el constante ataque de insectos herbívoros.

\section{Regeneración del bosque e interacciones biológicas}

Una de las metas en el campo de la ecología de comunidades es la de entender cómo es que algunas comunidades contienen un gran número de especies, mientras que otras 


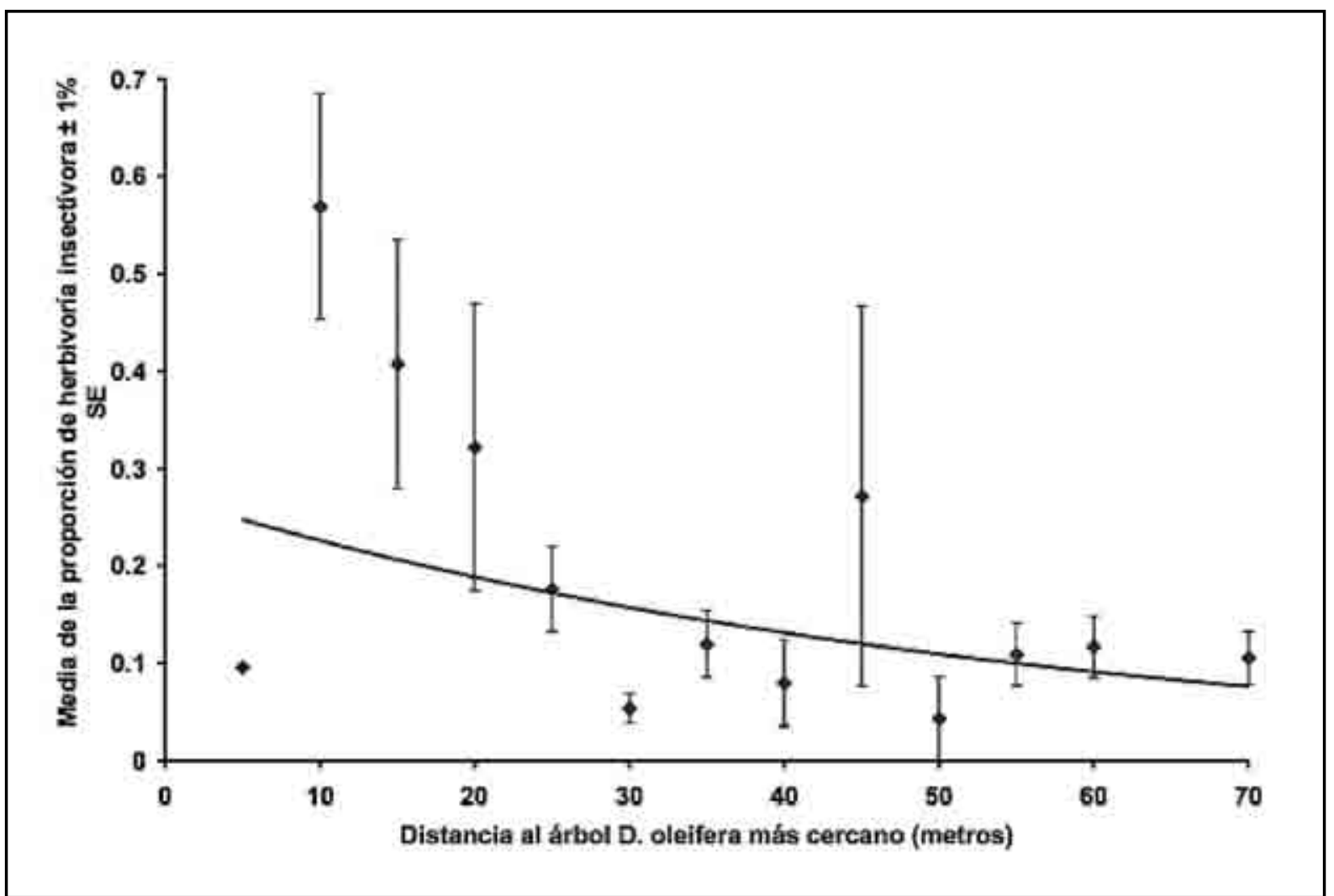

Figura 5. Media ponderada del daño de herbivoría insectívora sobre plántulas de D. oleifera como función dependiente de la distancia al árbol congénere más cercano (Ruiz et al. 2009a).

solo unas pocas. Una pregunta surge de la paradoja que mientras algunos organismos que parecieran tener nichos similares, y así deberían de sucumbir a las expectaciones de la teoría de exclusión competitiva, estas especies parecieran coexistir a través del tiempo (Hutchinson 1961). Las comunidades de plantas en un bosque húmedo tropical son ejemplos en los cuales se observan cientos de especies de árboles coexistiendo en un área determinada, mientras que en bosques templados, la misma área contiene típicamente solo 10 ó 20 (Stevens 1992). La pregunta se ha convertido en el foco de mucha investigación que busca la forma de detectar y entender los mecanismos responsables de tan alta biodiversidad (Chave et al. 2002; Connell 1978; Gilbert et al. 2001; Hubbell \& Foster 1986; Hubbell et al. 1999; Hurtt \& Pacala 1995; Tilman 1994).

Se ha considerado que entre los múltiples factores responsables del mantenimiento de la diversidad de especies están los disturbios. El papel de los disturbios periódicos como fuerzas que organizan los ecosistemas ha sido reconocido desde hace mucho tiempo (Connell 1978; Mooney y Gordon 1983; Pickett y White 1985). La idea fundamental es conocida como la "hipótesis de los disturbios intermedios". Esta idea propone que mientras las especies con nichos ecológicos similares podrían excluirse competitivamente unas a otras, si ocurren disturbios con regular frecuencia, éstos reiniciarían las poblaciones a sus densidades iniciales. En estas condiciones, el proceso de competición ecológica no permitirá que unas especies excluyan a otras (Connell 1978; Huston 1979). Los disturbios en bosques húmedos tropicales van desde la formación de un claro de luz, formado cuando un árbol cae en un bosque de copa cerradas (Brokaw 1985), hasta los daños masivos causados por tormentas tropicales (Yih et al. 1991). Ya que los huracanes, tifones y ciclones ocurren con regular frecuencia en áreas tropicales, sus influencias sobre la composición y estructura de bosques podría ser de particular importancia (Lawrence et al. 1991; Vandermeer et al. 2001). Dado el marco temporal de la demografía de bosques húmedos tropicales (cientos a miles de años), inclusive bajas frecuencias de ocurrencia de huracanes podrían considerarse como eventos comunes en la vida de un árbol.

Un huracán es un tipo de disturbio muy importante para el mantenimiento de la diversidad de los bosques húmedos tropicales (Ruiz et al. 2001; Vandermeer et al. 2000). El bosque del sureste de Nicaragua fue azotado por el huracán Juana en 1988, un huracán de categoría 4 (Cortez y Fonseca 
1988; Yih et al. 1991). Antes del huracán Juana, el bosque del sureste de Nicaragua presentaba características de bosque maduro, en vías de ser dominado por unas pocas especies en su copa. El paso del huracán Juana creó las condiciones de luz requeridas para la regeneración de muchas especies de árboles (Granzow-de la Cerda et al. 1997), propiciando altas tasas de crecimiento de especies que de otra forma hubieran sido suprimidas bajo la copa de bosque maduro (Ruiz et al. 2009c). La regeneración de árboles desde niveles inferiores del bosque hasta la copa ha sido al azar en relación a la identidad de especie (Vandermeer et al. en preparación); de esta forma, los disturbios contribuyen a mantener números altos de especies en bosques húmedos tropicales.

Después del huracán Juana, la especie D. oleifera resistió y se regeneró a partir de la reproducción de sus individuos adultos, aunque tomaron varios años para que la especie recuperara su capacidad reproductiva anterior al huracán. Iniciamos nuestras observaciones de la dispersión de semillas y distribución de plántulas de $D$. oleifera en el año 2000. A partir del 2002 notamos producciones de semillas muy altas en el sitio de estudio. El tiempo que tomó a los individuos adultos de D. oleifera en recuperar sus copas y ser capaces de producir cantidades considerables de semillas es de 10 a 15 años. Aunque en otras localidades más cercanas al punto donde el ojo del huracán impactó la costa, los individuos adultos de $D$. oleifera fueron más dañados y su producción de semillas es muy baja (observación personal). Sí el huracán Juana no hubiera impactado el bosque del sureste de Nicaragua, las especies típicas de bosque maduro, como $D$. oleifera, hubieran mantenido una alta productividad y dispersión de semilla, lo cual, en un bosque es crucial para establecerse en la copa. De esta forma, el número de especies en la copa de bosque no huracanado sería muy bajo, tal y como se ha observado en un bosque no huracanado del sureste de Nicaragua (Vandermeer et al. 2000).

\section{Conclusión}

Los resultados presentados muestran que la dispersión primaria y secundaria determina el proceso de reclutamiento en la especie de bosque húmedo tropical $D$. oleifera. La sobrevivencia de plántulas es mayor lejos del árbol congénere más cercano, donde el daño foliar es menor. La presencia de arañas podría estar contribuyendo al patrón de sobrevivencia de plántulas lejos del árbol congénere más cercano, puesto que las arañas podrían estar depredando a insectos herbívoros -una línea de investigación que amerita ser estudiada con mayor detalle. Las distribuciones espaciales del reclutamiento de plántulas ocurren en pequeñas agrupaciones localizadas lejos del árbol de $D$. oleifera. Este patrón de distribución podría ser cada vez más conspicuo debido al efecto de reducción de herbivoría insectívora por medio de las arañas. Entonces es necesario determinar hasta qué punto un incremento en el número de las plántulas atrae una cantidad de arañas que reduzca la magnitud del efecto negativo de insectos herbívoros. La regeneración de $D$. oleifera es el resultado de dispersión primaria y secundaria de semillas, herbivoría insectívora y presencia de arañas, en el sureste de Nicaragua.

Las investigaciones presentadas aquí abren nuevas y fascinantes líneas de investigación en el campo de la ecología de la dispersión y reclutamiento de plantas de bosque húmedo tropical. La cuantificación de estas interacciones biológicas servirá para desarrollar planes de conservación y uso adecuado de especies de los bosques húmedos tropicales. La próxima fase de investigación se está planeando sobre las bases académicas ya establecidas. Los objetivos del estudio serán: 1) la investigación de campo y la modelación estadística y matemática para tratar de responder preguntas de investigación que surgieron durante el proceso investigativo planteado en este articulo. 2) El acompañamiento intelectual a alumnos de pregrado, técnicos locales e investigadores formados por las iniciativas académicas presentadas y planteadas aquí.

\section{Recomendaciones}

La investigación como parte integral de un modelo universitario progresista podría conllevar, a mediano plazo, a la formación de más profesionales capaces de plantear, formular, diseñar y llevar a cabo iniciativas novedosas en los campos de investigación y educación. De 2005 a 2006 se canalizaron fondos de esta investigación para la realización de tertulias científicas en las cuales se discutieron tópicos relacionados con las áreas afines a los tópicos de tesis de los estudiantes de pregrado. Una meta alcanzada y que se recomienda continuar es la articulación de las tertulias científicas a Jornadas Científicas a nivel local y nacional. Se recomienda continuar los hermanamientos de universidades locales con universidades extranjeras lo cual ha rendido, como frutos, muchos profesionales capaces en las Regiones de la Costa Atlántica de Nicaragua.

\section{Agradecimientos}

El apoyo financiero fue gracias al auspicio de un Block Grant por parte de Departamento de Ecología y Biología Evolutiva, Universidad de Michigan (UM) a Javier Ruiz (JR), una beca del International Institute (UM) a JR, la beca (D-3939-1) del International Foundation for Science a JR y, la beca Rackham 
International Student Grant (UM) to JR. Agradecemos la fundación "Idea Wild" por la donación de una computadora portátil a JR para el desarrollo de esta investigación. NSF Research in Undergraduate Institutions (RUI) beca DEB0135350 a Douglas H. Boucher. Gracias a John Vandermeer, Ivette Perfecto, Iñigo Granzow de la Cerda, Beverly Rathcke, Cherryl Ingram, Sandra Yap y Paul Foster por sus valiosos comentarios durante el proceso científico de esta serie de investigaciones. Agradecimientos especiales al grupo de investigadores del Proyecto Biodiversidad-URACCAN por su apoyo en muchas formas.

\section{Anexo 1. Logros académicos al sistema universitario de} las Regiones Autónomas de las Costa Atlántica del Sureste de Nicaragua. Por medio de la presente investigación se contribuyó al desarrollo intelectual e investigativo de dos universidades públicas. A partir de la metodología de giras de campo se logró capacitar a aproximadamente 70 estudiantes locales en temáticas de Ecología Tropical, Biología de la Conservación, muestreo de campo, entrada de información digital y análisis estadístico. Debido a que cinco estudiantes sobresalieron académicamente se requirió formular proyecto para la localización de fondos que permitieran brindar el apoyo académico y financiero para sus investigaciones de pregrado. La canalización de los fondos se realizó a través de URACCAN. Cuatro estudiantes culminaron sus tesis de investigación teniendo como temas de investigación dispersión de semillas, los efectos de herbivoría sobre la regeneración de plántulas, catálogos taxonómicos de palmas y estudio de hormigas como bioindicadoras de uso y manejo de sistemas agroforestales. Dos estudiantes obtuvieron menciones honoríficas en las Jornadas Científicas impulsadas por el Consejo Nacional de Universidades de Nicaragua. Las tertulias científicas impartidas por mi grupo de investigación han sido la vanguardia científica en Ecología Tropical en Nicaragua. Los resultados presentados aquí han sido publicados en revistas científicas internacionales, de esta forma los resultados han contribuido al desarrollo de las ciencias naturales a nivel mundial. Como parte del desarrollo intelectual, los estudiantes formados se encuentran enseñando en las universidades o trabajando en instituciones locales.

Anexo 2. Material metodológico suplementario. Protocolos metodológicos necesarios para duplicar la serie de investigaciones presentadas en este artículo científico pueden encontrarse en el siguiente vínculo: http://deepblue. lib.umich.edu/bitstream/2027.42/60714/1/jeruiz_1.pdf

\section{Bibliografía}

Adams, P. 1983. "The theory of limiting similarity. Annu. Rev. Ecol. Syst. 14: 359-376.

Blundell, A. G., y D. R. Peart 1998. Distance-dependence in herbivory and foliar condition for juvenile Shorea trees in Bornean dipterocarp rain forest. Oecologia 117: 151-160.

Brokaw, N. V. L. 1985. Gap-Phase Regeneration in a Tropical Forest Ecology 66(3): 682-687.

Chave, J. H., H. C. Muller-Landau, y S. A. Levin 2002. Comparing classical community models: theoretical consequences for patterns of diversity. The American Naturalist 159(1): 1- 23.

Carpenter, S. R., J. J. Cole, J. R. Hodgson, J. F. Kitchell, M. L. Pace, D. Bade, K. L. Cottingham, T. E. Essington, J. N. Houser, y D. E. Schindler 2001. Trophic cascades, nutrients, and lake productivity: whole-lake experiments. Ecological Monographs 71(2): 163-186.

Clark, D. B., y D. A. Clark 1985. Seedling dynamics of a tropical tree: impacts of herbivory and meristem damage. Ecology 66(6): 1884-1892.

--- 1987. Population ecology and microhabitat distribution of Dipteryx panamensis a neotropical rain forest tree. Biotropica 19(3): 236-244.

Clark, D. B., D. A. Clark, y P. M. Rich 1993. Comparative analysis of micohabitat utilization by saplings of nine tree species in neotropical rain forest. Biotropica 25(4): 395-407.

Clark, J. S., B. Beckage, P. Camill, B. Cleveland, J. HilleRisLambers, J. Lichter, J. McLachlan, J. Mohan, y P. Wyckoff 1999a. Interpreting recruitment limitation in forests. American Journal of Botany 86: 1-16.

Clark, J. S., E. Macklin, y L. Wood 1998. Stages and spatial scale of recruitment limitation in southern Appalachian forests. Ecological Monographs 68(2): 213-235.

Clark, J. S., M. R. Silman, R. Kern, E. Macklin, y J. Hille-Ris-Lambers 1999b. Seed dispersal near and far: patterns across temperate and tropical forests. Ecology 80(5): 1475-1494.

Coley, D., y J. Barone 1996. Herbivory and plant defenses in tropical forest. Annu. Rev. Ecol. Syst. 27: 305-335.

Coley, P. D. 1983. Intraspecific variation in herbivory on two tropical tree species. Ecology 64(3): 426-433.

--- 1990. Tasas de herbivorismo en diferentes árboles tropicales. E.G. Leigh Jr., A.S. Rand, y D.M. Windsor (eds) En: 
Ecología de un bosque tropical: Ciclos estacionales y cambios a largo plazo. Instituto Smithsonian de Investigaciones Tropicales, Panama, Panama.: pp. 191-199.

Coley, P. D., J. P. Bryant, y F. S. I. Chapin 1985. Resource availability and plant antiherbivore defense. Science 230: 895-899.

Condit, R., P. S. Ashton, P. Baker, S. Bunyavejchewin, S. Gunatilleke, N. Gunatilleke, S. P. Hubbell, R. B. Foster, A. Itoh, J. V. LaFrankie, H. S. Lee, E. Losos, N. Manokaran, R. Sukumar, y T. Yamakura 2000. Spatial patterns in the distribution of tropical tree species. Science 288(1): 414-1418.

Condit, R., S. P. Hubbell, y R. B. Foster 1992. Recruitment near conspecific adults and the maintenance of tree and shrub diversity in a neotropical forest. The American Naturalist 140(2): 261-286.

Connell, J. H. 1971. On the roles of natural enemies in preventing competitive exclusion in some marine animals and in rain forest. Proceedings of the Advanced Study Institute on Dynamics of Numbers in Population, Oosterbeek, 1970 (eds. P. J. Den Boer y G. R. Grandwell)(Center of Agricultural Publishing and Documentation, Wageningen, The Netherlands.): pp. 298-312.

--- 1978. Diversity in tropical rain forest and coral reefs. Science 199: 1302-1310.

Cortez, G., y R. Fonseca 1988. El Ojo Maldito. Editorial Nueva Nicaragua. Managua, Nicaragua: 251 pp.

DeMattia, E. A., L. M. Curran, y B. J. Rathcke 2004. Effects of small rodents and large mammals on neotropical seeds. Ecology 85(8): 2161-2170.

Dirzo, R. 1987. Estudio sobre interacciones planta herbívoros en Los “Tuxtlas” Veracruz. Revista Biología Tropical 35: 119-132. Floren, A., A. Biun, y K. E. Linsenmair 2002. Arboreal ants as key predaors in tropical lowland forest trees. Oecologia 131: 137-144.

Forget, P.-M., y D. Wenny 2005. How to elucidate seed fate? a review of methods used to study seed removal and secondary seed dispersal. In: Seed fate predation, dispersal and seedling establishment. CABI Publishing. Edited by P.-M. Forget, J. E. Lambert, P. E. Hulme y S. B. Vander Wall: pp. 379-393.

Gilbert, G. S., K. E. Harms, D. N. Hamill, y S. P. Hubbell 2001. Effects of seedling size, El Niño drought, seedling density, and distance to nearest conspecific adult on 6-year survival of Ocotea whitei seedlings in Panamá. Oecologia 127: 509-516.

Granzow-de la Cerda, I., N. Zamora, J. H. Vandermeer, y D. H. Boucher 1997. Biodiversidad de especies arbóreas en el bosque tropical húmedo del Caribe Nicaragüense siete años después del huracán Juana. Revista de Biología Tropical (Costa Rica) 45(4): 1409-1419.

Gruner, D. S. 2004. Attenuation of top-down and bottom-up forces in a complex terrestrial community. Ecology 85: 3010-3022.

Hairston, N. G., E. S. Frederick, y B. S. Lawerence 1960. Community structure, population control, and competition. The American Naturalist 94: 421-425.

Holdridge, L. R. 1992. Ecología basada en zona de vida. Instituto Interamericano de Cooperación para la Agricultura. San José, Costa Rica.

Howe, H. F., y J. Smallwood 1982. Ecology of seed dispersal. Ann. Rev. Ecol. Syst. 13: 201-228.

Hubbell, S. P. 2001. The Unified Neutral Theory of Biodiversity and Biogeography. Monographs in Population Biology 32 Princeton University Press, Princeton, NJ: pp. 375.

Hubbell, S. P., R. B. Foster, S. T. O’Brien, K. E. Harms, R. Condit, B. Wachsler, S. J. Wright, y S. Loo de Lao 1999. Lightgap disturbances, recruitment limitation, and tree diversity in a neotropical forest. Science 283: 554-557.

Hurtt, G. C., y S. W. Pacala 1995. The consequences of recruitment limitation: reconciling chance, history and competitive differences between plants. Journal of Theoretical Biology 176: 1-12.

Hubbell, S. P., y R. B. Foster 1986. Biology, chance and history and the structure of tropical forest tree communities. Community Ecology (eds. J. Diamond \& T. J. Case). Harper \& Row, New York: pp. 314-329.

Hutchinson, G. E. 1961 The paradox of the plankton. American Naturalist 95: 137-145.

Hurtt, G. C., y S. W. Pacala 1995. The consequences of recruitment limitation: reconciling chance, history and competitive differences between plants. Journal of Theoretical Biology 176: 1-12.

Huston, M. 1979. A general hypothesis of species diversity. American Natrualist 113: 137-145.

Hyatt, L. A., M. S. Rosenberg, T. G. Howard, G. Bole, W. Fang, K. Anastacia, K. Brown, R. Grella, K. Hinman, J. P. Kruidiziel, y J. Gurevitch 2003. The distance dependence prediction of the Janzen-Connell hypothesis: a meta-analysis. Oikos 103: 590-602.

Janzen, D. H. 1970. Herbivores and the number of tree species in tropical forest. The American Natrualist 104: 501-528.

Lawrence, R. W., D. J. Lodge, y R. B. Waide 1991. An introduction to hurricanes in the Caribbean Biotropica 23(a): 313-316.

Levins, R. 1974. Qualitative analysis of partially specified systems. Annals of the New York Academy of Science 231: 123-138.

Mooney, H. A., y M. Gordon 1983. Disturbance and ecosystem. Springer-Verlag. 
Moreau, G., E. S. Elveleigh, C. J. Lucarotti, y D. T. Quiring 2006. Ecosystem alteration modifies the relative strength of bottom-up and top-down forces in a herbivore population. Journal of Animal Ecology 75: 853-861.

Muller-Landau, H. C., J. Wright, O. Calderon, S. P. Hubbell, y R. B. Foster 2002. Assessing recruitment limitation: concepts, methods and case study from a tropical forest. CAB International. Seed Dispersal and Frugivory: Ecology, Evolution and Conservation (eds D.J. Levey, W. R. Silva y M. Galetti): 35-52.

Pickett, S. T. A., y P. S. White 1985. Natural distrubance and patchy dynamics. Academic Press, New York.

Reid, F. A. 1997. A field guide to the mammals of central america and southeast Mexico. Oxford University Press: pp. 334.

Ribbens, E., J. A. J. Silander, y S. W. Pacala 1994. Seedling recuitment in forests: calibrating models to predict patterns of tree sedling dispersion. Ecology 75(6): 1794-1806.

Romero, G. Q., y J. Vasconcellos-Neto 2004. Beneficial effects of flower-dwelling predators on their host plant. Ecology 85(2): 446-457.

Ruiz, J. 2008. Effects of dispersal and insect herbivory on seedling recruitment of Dipteryx oleifera Benth (Fabaceae) a tropical tree. Ph.D. dissertation. The University of Michigan.

Ruiz, J., D. Boucher, J. H. Vandermeer, I. Granzow-de-la-Cerda, I. Perfecto, y V. Martínez-Salgado 2001. Recuperación inicial del bosque incendiado y previamente afectado por el huracán Juana en Nicaragua. Encuentro 58: 66-75.

Ruiz, J., D. H. Boucher, C. L. Ingram-Flores, y L. F. Chaves 2009a. Beneficial effect of spider presence on seedling recruitment of the neotropical tree Dipteryx oleifera (Fabaceae). Revista de Biología Tropical 53: 000-000.

Ruiz, J., y H. D. Boucher 2009. Recruitment of Dipteryx oleifera Benth (Fabaceae) correlates with bat seed dispersal, rodent seed dispersal and roosting palm species away from conspecific trees. Brenesia 71: 000-000.

Ruiz, J., H. D. Boucher, D. Ruiz-Moreno, y C. Ingram-Flores 2009b. Recruitment dynamics of the tropical rainforest tree Dipteryx oleifera (Fabaceae) in Eastern Nicaragua. Revista de Biología Tropical 57(1-2): 321-338.

Ruiz, J., J. Vandermeer, I. Granzow de la Cerda, I. Perfecto, y H. D. Boucher 2008. Regeneración de bosques huracanados de Nicaragua (1988-2007). Wani 52: 6-16.

Ruiz, J. 2008. Prueba de las hipótesis de Janzen-Connell y de limitación de reclutamiento en el contexto de la organización de bosques húmedos tropicales. Wani 52: 22-33.

Ruiz, J. 2009. Plant Recruitment Limitation. Effects of Dispersal and Arthropod Herbivory on Seedling Recruitment of Dipteryx oleifera (Fabaceae) a Tropical Tree. Verlag Dr. Müller Publishing House. Germany. ISBN 978-3-639-19765-5

Ruiz, J., J. Vandermeer, I. Granzow-de-la-Cerda, H. D. Boucher, e I. Perfecto 2009c. Growth and mortality patterns in the thinning canopy of a post-hurricane rainforest in eastern Nicaragua (1990-2005). Revista de Biología Tropical: En revisión.

Sullivan, J. J. 2003. Density-dependent shoot-borer herbivory increases the age of first reproduction and mortality of neotropical tree saplings. Oecologia 136: 96-106.

Stevens, G. C. 1992. The elevation gradient in altitudinal range: an extension of rapoport's latitudinal rule to altitude. The American Naturalist 140(6): 893-911.

Tilman, D. 1994. Competition and biodiversity in spatially structured habitats. Ecology 75(1): 2-16.

Van Bael, S. A., y J. D. Brawn 2005. The direct and indirect effects of insectivory by birds in two contrasting Neotropical forest. Oecologia 145: 658-668.

Van Bael, S. A., J. D. Brawn, y S. K. Robinson 2003. Birds defend trees from herbivores in neotropical forest canopy. PNAS 100(14): 8304-8307.

Vandermeer, J., I. Granzow-de-la-Cerda, H. D. Boucher, I. Perfecto, y J. Ruiz en preparación. Tree mortality in a thinning phase-canopy of a Nicaraguan hurricane forest.

Vandermeer, J., I. Perfecto, G. Ibarra-Nunez, S. Phillpott, y A. Garcia-Ballinas 2002. Ants (Azteca sp.) as potential biological control agents in shade coffee production in Chiapas, Mexico. Agroforestry Systems 56: 271-276.

Vandermeer, J. H., D. H. Boucher, I. Granzow-de-la-Cerda, y I. Perfecto 2001. Growth and development of the thinning canopy in a post-hurricane tropical rain forest in Nicaragua. Forest Ecology and Management 148: 221-242.

Vandermeer, J. H., I. Granzow-de-la-Cerda, D. H. Boucher, I. Perfecto, y J. Ruiz 2000.

Vander Wall, S. B., K. M. Kuhn, and M. J. Beck 2005. Seed removal, seed predation, and secondary dispersal. Ecology 86(3): 801-806.

Hurricane disturbance and tropical tree species diversity. Science 290: 788 -791.

Yih, K., D. H. Boucher, J. H. Vandermeer, y N. Zamora 1991. Recovery of the rainforest of southeastern Nicaragua after destruction by hurricane Joan. Biotropica 23: 106-113. 